\title{
Téoros
}

Revue de recherche en tourisme

\section{Le tourisme gourmand}

La dictature des guides?

\section{Normand Cazelais}

Volume 25, numéro 1, printemps 2006

Entre la culture du goût et le goût de la culture

URI : https://id.erudit.org/iderudit/1071028ar

DOI : https://doi.org/10.7202/1071028ar

Aller au sommaire du numéro

Éditeur(s)

Université du Québec à Montréal

ISSN

0712-8657 (imprimé)

1923-2705 (numérique)

Découvrir la revue

Citer cet article

Cazelais, N. (2006). Le tourisme gourmand : la dictature des guides ? Téoros, 25(1), 15-18. https://doi.org/10.7202/1071028ar

Ce document est protégé par la loi sur le droit d'auteur. L'utilisation des services d'Érudit (y compris la reproduction) est assujettie à sa politique d'utilisation que vous pouvez consulter en ligne.

https://apropos.erudit.org/fr/usagers/politique-dutilisation/
Cet article est diffusé et préservé par Érudit.

Érudit est un consortium interuniversitaire sans but lucratif composé de l'Université de Montréal, l'Université Laval et l'Université du Québec à Montréal. Il a pour mission la promotion et la valorisation de la recherche. https://www.erudit.org/fr/ 


\section{Le tourisme gourmand La dictature des guides?}

\section{Normand Cazelais}

Le triangle tourisme, gastronomie et information est riche d'enseignements. On connaît notamment l'influence qu'ont eue et qu'ont toujours les critiques gastronomiques sur la "culture gourmande ${ }^{1}$ de la France. En ce pays comme ailleurs, ils sont tout à la fois redoutés et enviés. Par l'entremise des guides et des magazines spécialisés ou par les médias d'information générale, ils agissent sur le comportement des clients et des établissements, entraînant chez les premiers des modifications de leurs fréquentations et même de leurs goûts, poussant les seconds à vouloir élargir leur notoriété et adopter, en conséquence, des stratégies de nature à se concilier les faveurs de ces « experts" et, ultimement, celles de la clientèle.

La réflexion pourrait, mutatis mutandis, s'appliquer aux chroniqueurs de tourisme, comme l'illustre l'histoire des guides culinaires qui sont aussi des guides de voyage.

\section{Un peu d'histoire}

Les guides gastronomiques existent en plusieurs langues et pour de nombreuses destinations. Retenons ceux d'Egon Ronay pour la Grande-Bretagne et d'Harry Rolnik pour la Chine et les pays du Sud-Est asiatique, la collection Ristoranti de Luigi Veronelli pour l'Italie, les Zagat Restaurant Survey et les Epicurean Rendezvous pour les États-Unis. Les balbutiements des guides de voyage remontent aussi loin que les anciennes dynasties chinoises ou les civilisations mésopotamienne et égyptienne.

Écrit en 1519 à l'intention des pèlerins qui se dirigeaient vers la Terre sainte, le Viaggio da
Venezia donnait des conseils à ces voyageurs de l'infini qui vivaient des conditions de déplacement à tout le moins précaires : marcher par des chemins incertains, aller à dos de cheval, de mulet ou de bœuf, s'entasser sur des navires chargés de marchandises (et de rats), dormir à la belle étoile ou dans des coupe-gorge déguisés en auberge, subir guerres, taxes et vexations, risquer à tout moment de se perdre, éviter les maladies, les épidémies, la faim et la soif qui guettaient, se heurter aux problèmes de langue, ne pas avoir la certitude de revenir ni de retrouver dans le même état ce qui avait été laissé derrière. À cette époque, le Viaggio da Venezia et les autres documents similaires ne parlaient guère des ressources culinaires des régions traversées ni de leurs bonnes tables...

Faut-il s'en étonner? Le premier guide des restaurants, l'Almanach des gourmands, a vu le jour en France en 1804. Depuis, rappelle l'étatsunien Daniel Rogov (n.d.), "gastronomes, restaurateurs and chefs have had a mixed collection of love and hate reactions for such publications". Le premier guide moderne des restaurants, le Michelin, a paru, quant à lui, en 1900. Avant de devenir la "bible de la critique gastronomique ", ce n'était qu'un petit carnet de poche - rouge, bien sûr - d'une vingtaine de pages; il était alors « offert gracieusement aux chauffeurs » audacieux, mais de plus en plus nombreux, qui s'aventuraient sur des routes pleines de cahots et de pièges au volant de leur véhicule automobile que la compagnie de Clermont-Ferrand espérait équiper de ses pneumatiques.

Bourré de renseignements pratiques, le Michelin offrait, à travers l'Hexagone, des adresses de mécaniciens, de dépôts de carburant et d'établissements " permettant de se loger et de se nourrir ». Avec les ans, il a évolué pendant que l'automobile s'imposait comme un instrument de liberté et que vacances et loisirs se démocratisaient, de telle sorte que la table et ses «plaisirs » ont pris de plus en plus d'importance en ses pages qui dépassent aujourd'hui le millier. Ce fut en 1931, une trentaine d'années après sa naissance, que les éditeurs ont envisagé de recourir à un système de classification marqué de un à trois macarons - les fameuses " étoiles »- pour identifier les meilleures tables.

\section{Michelin certes, mais aussi d'autres guides}

Le Michelin, qui a fait et défait les réputations et les fortunes de générations de cuisiniers, démontre à lui seul le pouvoir de l'information

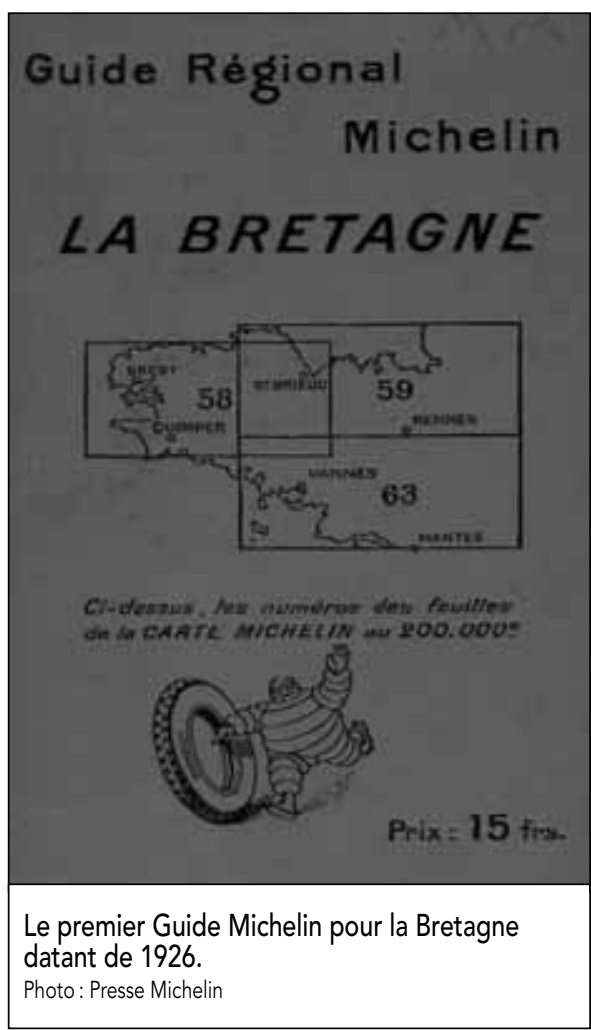


dans les mondes de la gastronomie et du tourisme. Combien de voyageurs, en effet, ont parcouru et découvert les diverses régions de la France - et d'autres pays, par la suite- en se fiant aux recommandations du guide rouge?

Ne retenons qu'un cas, celui de la ville de Roye. Voici comment Le Petit Robert des noms propres (2004) décrit cette inconnue: "Chef-lieu de canton de la Somme, 8529 habitants. Église à chœur gothique reconstruite après la Première Guerre mondiale. Sucrerie, marché de grains, industries diversifiées." Le site Internet de la ville ${ }^{2}$ retient toutefois d'autres éléments, dont ce grand titre d'introduction: "Roye, cité gastronomique et seule ville 'étoilée' du département de la Somme depuis 104 ans! » De toute évidence, les autorités municipales s'enorgueillissent d'avoir eu, dès la première édition, un établissement, à savoir l'Hôtel du Commerce, qui figurait dans le Michelin... même si les «étoiles » n'existaient pas à ce moment-là. À leurs yeux, le Michelin a confirmé la vocation de cette "ville-relais réputée " sur la route des Flandres: "Roye a maintenu au fil des siècles cette réputation et aujourd'hui les grands guides gastronomiques soulignent à travers leurs pages la qualité de ses établissements. " À l'appui de cette affirmation, le site étale les pages couvertures du Michelin, des guides Pudlowski et

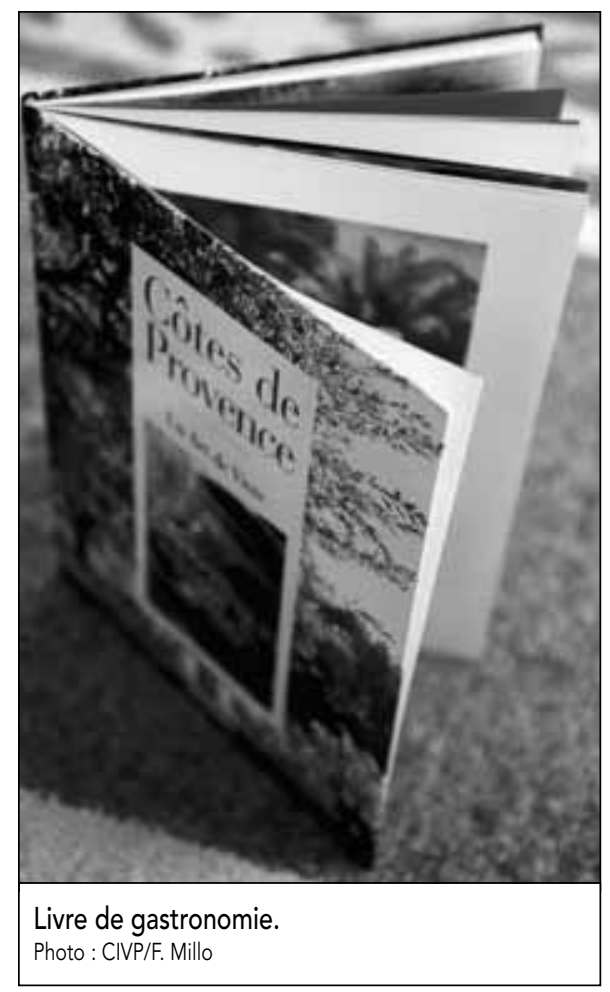

Champérard, du Bottin Gourmand, du Petit Futé et du GaultMillau, avant d'ajouter : "Si vous avez connaissance d'autres articles concernant les hôteliers-restaurateurs de Roye, n'hésitez pas à nous les communiquer. » L'invitation parle d'elle-même...

Toute reconnaissante qu'elle soit de la notoriété que lui a procurée le Michelin, Roye ne passe pas sous silence, comme on le voit, la contribution d'autres guides associant voyage et gastronomie. Car il y a maintenant des décennies que le guide rouge Michelin a des concurrents. Et, au premier rang, le GaultMillau qui, à sa manière, a refaçonné le visage tant touristique que culinaire de la France.

Critiques gastronomiques de métier, Henri Gault et Christian Millau ont été les propagateurs les plus connus de ce qu'on a appelé voici trois décennies la «nouvelle cuisine française ", caractérisée par la mise au rancart de sauces "stéréotypées » et par un retour à la saveur "fondamentale » des aliments. Paul Bocuse à Lyon, les frères Troisgros à Roanne, Michel Guérard à Eugénie-les-Bains et Alain Senderens à Paris sont les chefs les plus célèbres associés à ce mouvement culinaire. En unissant leurs efforts, Gault et Millau ont ouvert de nouvelles voies et, même s'ils se sont séparés et retirés par la suite, la marque de commerce demeure.

Beaucoup de professionnels, d'amateurs de fine cuisine et de voyageurs reprochent au Michelin son conservatisme, l'accusant d'être lent à accorder des macarons - donc à reconnaître le mérite et le talent - et d'être encore plus lent à les retirer. Selon André Gayot, un de leurs associés de la première heure, Gault et Millau ont jeté un lourd pavé dans la mare en fondant Le Nouveau Guide en mars 1969 : ils attaquaient de front l'establishment culinaire français et son plus illustre défenseur. Dans la foulée des événements de mai 1968 et des Cahiers du cinéma dont les animateurs - Truffaut, Godard, Malle, Renais, entre autres - allaient donner vie à une "nouvelle vague " qui a secoué toute l'industrie du septième art, ils proclamaient haut et fort que la société avait changé et qu'elle avait besoin d'une « révolution ».

Leur coup de génie fut de substituer à la rigide échelle des macarons du Michelin une notation plus souple - et qualifiée par certains de plus subtile -, s'étendant de 0 à 20 points, calquée sur celle en vigueur dans toutes les écoles et tous les lycées de la République, donc familière à tout un chacun. Qui plus est, cette notation voulait privilégier l'innovation, l'esprit créatif et le retour aux sources du goût ; elle ne tenait compte, dans son évaluation, que de la qualité de la cuisine et des plats et non du service, du décor, de l'atmosphère des lieux ou de l'accueil, sans toutefois négliger ces aspects dans les commentaires complémentaires. Ce qui tombait bien, car les compatriotes de Gault et Millau et les visiteurs de la France en général voulaient en apprendre davantage sur les restaurants que ne leur en disait le Michelin. "Nos voyages à travers tout le pays, raconte André Gayot (n.d.), nous ont permis de découvrir sans cesse des restaurants et des bistros où de jeunes chefs ouvraient la voie à une nouvelle gastronomie française. Notre rôle a été de fédérer en quelque sorte ces tendances, de mettre en contact ces pionniers les uns avec les autres, de diffuser leurs façons de faire et de penser. Et de parler de nos découvertes.»

Ce que confirme Jacques Pépin ${ }^{3}$ dans son ouvrage, The Apprentice (2003), en ajoutant :

Nouvelle cuisine was soon popularized by food writers eager to discover something new, and by journalists who overpraised it. It became the rage, a new creed, and the intellectuals of the movement were the French journalists Gault and Millau. They not only extolled this new way of cooking but set down the cuisine's bylaws with the help of some of the great chefs of France.

Une nouvelle géographie culinaire était née. Et, du coup, le tourisme français recevait un influx très puissant dont les effets se font encore sentir.

Cette nouvelle cuisine, largement associée à une cuisine allégée dite "minceur », a aussi connu ses détracteurs : on a notamment reproché à plusieurs chefs et maisons qui s'en réclamaient de réduire les assiettes à des portions congrues tout en soufflant de façon indue les prix vers le haut ${ }^{4}$. Ces nouvelles toques, a relevé le gastronome David Rosengarten dans son livre de recettes It's All American Food (2003), "forged nothing less than a revolution, showing the world that French technique and new, modern culinary ideas were not incompatible ». Plus encore, soutient-il, ils ont ex- 


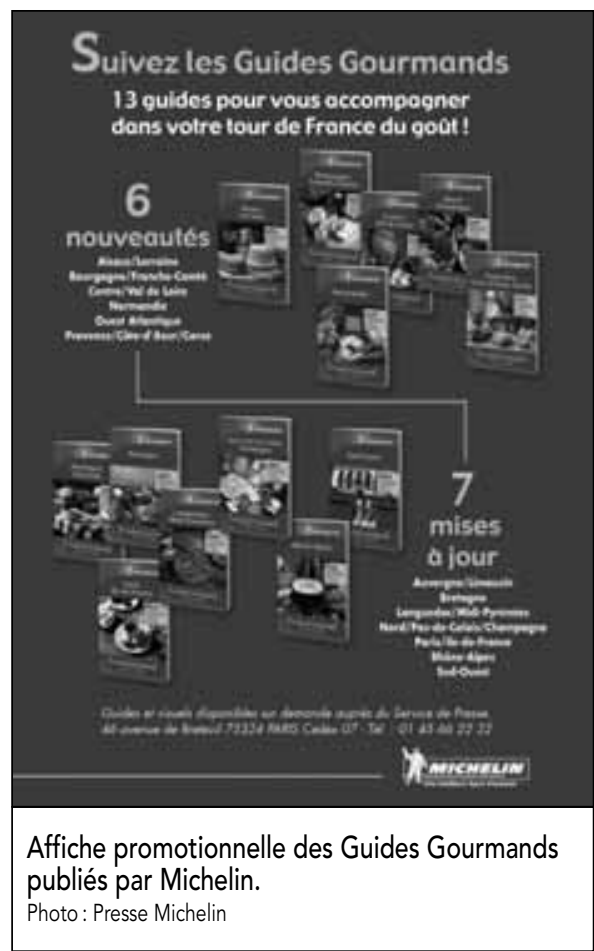

porté leur "revolution » : "It swept the entire world, beginning with the U.S. America was particularly receptive, because it is always open to innovation and also because gastronomy was then a barren land. American chefs jumped on the concept and 'Nouvelle Cuisine' oriented restaurants started to flourish in New York.» Grâce également, aurait-il pu préciser, au support enthousiaste d'une presse gastronomique et touristique qui a favorisé pareille expansion.

Les années ont passé. «What does Nouvelle Cuisine mean today? ", demandait Rosengarten, en enchaînant une réponse de son cru :

[...] the revolution has transformed [it] into an institution. From Vancouver to Marseille and from London to Tel Aviv, we all eat in a far different way than our parents did. Nouvelle Cuisine has literally revolutionized the way food is absorbed worldwide, in the comparable layers of societies, of course.

Dans L'escargot, bulletin de l'association Slow Food de France, Roger Feuilly (2000), président du regroupement de restaurants Convivium Paris, établissait un bilan différent :

Voilà déjà une quinzaine d'années, il était de bon ton - du temps de la nouvelle cuisine française finissante - de crier haro sur "Les assassins de la cuisine française ", titre alarmant du Nouvel Observateur d'un mois d'août 1985. [...] Et l'hebdomadaire de prédire, avec bien d'autres que, sous l'influence conjuguée (grande, il faut en convenir à l'époque) de messieurs Gault et Millau, de la pression fiscale et de l'industrialisation alimentaire, que la bistrologie française allait disparaître. [...] Qu'en est-il aujourd'hui ? De ces prédictions, il ne reste plus grandchose. Gault a disparu récemment ${ }^{5}$, Millau s'est retiré depuis belle lurette. Leur magazine - vendu et revendu-, jadis mensuel, aujourd'hui bimestriel, mesure son influence à l'aune de la baisse constante de sa diffusion. Leur guide n'intéresse plus guère et ne fait plus, d'évidence et à l'inverse du Michelin, peur à personne. [...] La vérité, c'est qu'on n'a jamais aussi bien mangé qu'aujourd'hui. [...] Dans le même temps, la malbouffe va son train. Mais la résistance slowfoodienne s'organise, ici, ailleurs et maintenant pour que le beau et le bon se coulent chaque jour dans le moule de nos assiettes et dans nos verres.

\section{Et maintenant ?}

De toute évidence, les guides gastronomiques ont activement contribué à consolider sinon à développer la réputation gastronomique de la France, en faisant une destination particulièrement rêvée pour les amateurs de bonne chère. Dans la lignée de Carême, Brillat-Savarin, Grimod de La Reynière, Curnonsky et al., le Michelin a consacré les canons de la tradition qu'il a enchâssés en ses pages en «étoilant » des restaurants. Le GaultMillau, dès son lancement, a fortement contesté cette conception de la qualité, s'en prenant au conservatisme et à l'immobilisme du guide rouge et proclamant le droit à mettre au point une nouvelle cuisine dont Henri Gault a d'ailleurs défini le code et les règles dans un article retentissant en 1972. À chaque fois, le rôle de l'information a été primordial.

Par les temps qui courent, l'univers de la cuisine est envahi par des termes alarmistes référant à la mondialisation, aux techniques transgéniques, à la malbouffe ${ }^{6}$. À l'échelle de la planète, la chaîne McDonald's, par exemple, s'est transformée en un emblème à honnir. Et une autre révolution est en train de s'opérer, à l'instigation cette fois des Italiens : celle du slow food.
Citons encore une fois L'escargot (Slow Food, 2000) qui livre les grandes lignes de sa philosophie :

Slow Food est lente nourriture, vivre lent, ne pas revêtir une austère robe de bure. Toujours faire retentir l'hymne de la viticulture. Mettre les anchois en saumure, le saumon rosé en fumure, les petits rougets en friture. La chair d'agneau, savoir rôtir, en sauvegardant sa texture et toujours savoir ralentir. Slow Food est lente nourriture.

Sur un ton plus pragmatique, le texte explique:

L'Association Slow Food se voulait une association œnogastronomique, se souciant du plaisir des sens et du maintien de traditions gastronomiques de qualité. [...] Nous avons pris conscience de l'essentiel, de ce qui est le point de départ de l'action et de l'esprit même de notre mouvement: la préservation de la biodiversité. En train de devenir une véritable mouvement international ${ }^{7}$, Slow Food évolue ainsi de l'œnogastronomie vers l'écogastronomie, dans la mesure où il n'est pas raisonnable d'ignorer l'accès aux plaisirs du goût pour les générations futures.

Plus récemment, Jean Lhéritier (2005), président de Slow Food France, précisait dans Le Bulletin :

Nous proposons une définition nouvelle de la gastronomie, mieux articulée aux enjeux de l'alimentation d'aujourd'hui. [...] C'est un exercice périlleux. [...] La gastronomie, comme l'agriculture et comme le plaisir, c'est l'affaire de tous. [...] Slow Food, ce n'est pas un grand écart entre la petite production agricole et la cuisine des chefs étoilés, c'est la symbiose entre l'aspiration à manger mieux et le désir de produire mieux, dans l'espoir de respecter notre Terre Mère ${ }^{8}$.

Déjà, dans la presse spécialisée, qu'elle soit gastronomique ou touristique, se manifeste un réel intérêt, pour ne pas dire un engouement, pour cet autre appel à une "réinvention de la cuisine » qui se veut tout à la fois un retour aux sources et une résistance à l'uniformisation du goût et des produits de l'agriculture. Le monde de l'information est encore appelé à la rescousse pour en établir, par ses analyses, ses comp- 
tes rendus et ses témoignages, une reconnaissance "objective " et constituer, de la sorte, un contrepoids aux moyens " omnipuissants » de la cuisine et de la restauration que rejette le slow food.

Mais, cette fois-ci, l'utilisation des outils d'information est différente. De même que l'optique. II ne s'agit plus seulement du plaisir de bien manger conjugué au fait de produire des aliments de qualité et de coter des établissements et leurs chefs, il s'agit de poser un nouveau regard sur le monde par l'entremise de la gastronomie. Le guide ne s'appelle plus Michelin ou GaultMillau, mais L'escargot: ce véhicule, qui n'est pas un guide au sens convenu du terme, est distribué en une large variété de commerces et de points de vente ${ }^{9}$; il ne limite pas sa fonction à la seule cuisine des restaurants, mais l'élargit à l'ensemble de la gastronomie, depuis la culture agricole jusqu'à l'assiette et la bouche du consommateur. De la sorte, la notion et le rôle mêmes du guide s'en trouvent transformés.

Une autre dimension à ne pas négliger, celle de l'électronique. Michelin, GaultMillau et tous les guides qu'on pourrait qualifier de classiques, en français comme dans les autres langues, ont été diffusés sous format papier. Leur pouvoir a été en quelque sorte étroitement lié à la volonté des consommateurs - amateurs de bonne chère ou voyageurs - de payer pour se procurer physiquement les guides, les magazines ou les médias écrits en question. Déjà, la radio et la télévision ont contribué à changer la donne, permettant aux critiques et aux clients d'entrer en contact sans le support du papier et de l'écrit. Mais subsistait toujours l'impossibilité pour ces derniers d'exprimer directement et rapidement leurs opinions et leurs réactions.

La cybernétique, l'électronique et, en particulier, la toile du Web ont radicalement transformé ce contexte. L'univers du virtuel donne à tout individu, qu'il soit propriétaire d'un ordinateur ou qu'il utilise les services d'un fournisseur collectif (de type café Internet, par exemple), un accès direct et instantané aux critiques, aux lieux, aux établissements, aux chefs. De toute évidence, les rapports de force et d'influence s'en trouvent changés et continueront de l'être encore davantage. C'est ce qu'ont compris, on le voit bien, les initiateurs et les promoteurs du mouvement Slow Food.

\section{Encadré}

Un site Web, mis au point par l'Institut français de Madrid ${ }^{10}$, propose des Carnets de route pour les gourmands dans les grandes régions françaises : I'Île-de-France, le Nord, I'Alsace-Lorraine et la Bourgogne, la Normandie et la Bretagne, le Centre et l'Auvergne, la Provence et la Côte d'Azur, le Sud-Ouest. Pour chacune, le site présente une recette (ex. : celle de la blanquette de veau en Île-deFrance), des suggestions de plats à déguster (ex.: les bergamotes et la choucroute en Alsace), des textes à lire (ex.: Les trois messes basses d'Alphonse Daudet en Provence). Heureux mariage culturel de voyage, de gastronomie et d'information.

D'entrée de jeu, ces quelques lignes de Raymond Queneau (n.d.) annoncent des textes "succulents qui se goûtent, se savourent, se dévorent... »:

«Prenez un mot prenez-en deux faites-les cuir' comme des œufs prenez un petit bout de sens puis un grand morceau d'innocence faites chauffer à petit feu au petit feu de la technique versez une sauce énigmatique saupoudrez de quelques étoiles poivrez puis mettez les voiles [...]. »

Normand Cazelais est géographe et journaliste.

\section{Notes}

1 Lire à cet effet les réflexions d'Alain Fusion sur le site [http://www.lesrestos.com].

2 [www.roye80.fr], 2004.

3 Qualifié par la critique gastronomique Julia Child de "best chef in America", Jacques Pépin, qui a été le cuisinier attitré du général de Gaulle, anime des émissions culinaires sur le réseau PBS depuis plus de vingt ans.

4 Certains - et non les moindres - ont donné un nouveau cap à leur carrière. Ainsi, le magazine Le Lapin gourmand annonçait, le 23 restaurant Lucas Carton de la place de La Madeleine ", renonce à ses trois étoiles du Guide Michelin pour avoir plus de liberté et offrir une nouvelle convivialité dans un établissement plus sensuel, plus féminin et plus ouvert dès septembre 2005 et ce, à un coût raisonnable (moins de 100 euros). Lui-même s'en est expliqué dans les pages du Figaro Magazine (édition du 23 octobre 2005), en utilisant donc le levier et le pouvoir de la presse.

5 II est décédé le 9 juillet 2000.

6 Dans une chronique du quotidien La Presse (12 décembre 2005), le journaliste Pierre-
Paul Gagné demandait s'il ne fallait pas « taxer la malbouffe » en soulignant que « la mauvaise alimentation, particulièrement la malbouffe, va occuper une bonne partie du discours public au cours des prochaines années ". II poursuivait en citant diverses opinions de lecteurs, notamment celle d'un chercheur québécois en distribution et sécurité alimentaire : "L'industrie de la malbouffe est dorénavant vouée au même destin que l'industrie du tabac. Déjà lourdement pourfendue par nombre de législateurs publics et de consommateurs dans le monde, l'industrie de l'alimentation sera tenue de réformer ses stratégies de marketing à brève échéance. "

7 Fondé en 1989, Slow Food, qui compte 82000 adhérents et quelque 800 conviviums (unités locales) dans 50 pays, a son siège à Bra en Italie. Des associations nationales ont été établies en France, aux États-Unis, en Allemagne, en Suisse, au Japon et, depuis 2001, au Québec (à cet effet, consulter le site [www.slowfoodquebec.com]).

8 C'est dans cet esprit que se tiendra, du 26 au 30 octobre 2006, à Turin en Italie, la deuxième édition de Terra Madre (Salone del Gusto - Salon du goût), qui rassemblera plus de 5000 paysans et producteurs, de nombreux universitaires et quelque 1000 chefs. II s'agit de la plus grande manifestation internationale ayant trait aux vins et à la gastronomie.

9 Au Québec comme dans la plupart des pays où Slow Food affirme sa présence, des commerces affichent des logos du mouvement, tant pour témoigner de l'option de l'établissement que pour, en certains cas, accorder des réductions aux membres.

10 [www.ifmadrid.com].

\section{Bibliographie}

Feuilly, Roger (2000), "Mauvaise humeur d'hier, bonne humeur d'aujourd'hui ", L'escargot, vol. 1, p. 6-7.

Gayot, André (n.d.), "Of Stars and Tripes - The True Story of Nouvelle Cuisine ", article disponible sur le site [www.gayot.com].

Lhéritier, Jean (2005), Le Bulletin, Lettre mensuelle d'information de Slow Food France, Paris n ${ }^{\circ} 5$, novembre.

Pépin, Jacques (2003), The Apprentice: My Life in the Kitchen, Houghton Mifflin Company, New York.

Queneau, Raymond (n.d.), [http://www.ifmadrid .com/gourmands/ecrits/index.html].

Rogov, Daniel (n.d.), «A Mini-Guide to Restaurant Guides Judging the Judges ", article disponible sur le site [www.stratsplace.com/rogov].

Rosengarten, David (2003), It's All American Food: The Best Recipes for More Than 400 New American Classics, États-Unis, Library in Congress Cataloging-in-publication Data.

Slow Food, 2000, L'escargot, bulletin de l'association Slow Food de France. 